\title{
A SYMPLECTIC FIXED POINT THEOREM ON OPEN MANIFOLDS
}

\author{
MICHAEL COLVIN AND KENT MORRISON
}

\begin{abstract}
In 1968 Bourgin proved that every measure-preserving, orientationpreserving homeomorphism of the open disk has a fixed point, and he asked whether such a result held in higher dimensions. Asimov, in 1976, constructed counterexamples in all higher dimensions. In this paper we answer a weakened form of Bourgin's question dealing with symplectic diffeomorphisms: every symplectic diffeomorphism of an even-dimensional cell sufficiently close to the identity in the $C^{1}$-fine topology has a fixed point. This result follows from a more general result on open manifolds and symplectic diffeomorphisms.
\end{abstract}

Introduction. Fixed point theorems for area-preserving mappings have a history which dates back to Poincaré's "last geometric theorem", i.e., any area-preserving mapping of an annulus which twists the boundary curves in opposite directions has at least two fixed points. More recently it has been proved that any area-preserving, orientation-preserving mapping of the two-dimensional sphere into itself possesses at least two distinct fixed points (see [N, Si]). In the setting of noncompact manifolds, Bourgin [B] showed that any measure-preserving, orientation-preserving homeomorphism of the open two-cell $B^{2}$ has a fixed point. For Bourgin's theorem one assumes that the measure is finite on $B^{2}$ and that the measure of a nonempty open set is positive. Bourgin also gave a counterexample to the generalization of the theorem for the open ball in $\mathbf{R}^{135}$ and asked the question whether his theorem remains valid for the open balls in low dimensions. In [As] Asimov constructed counterexamples for all dimensions greater than two and actually got a flow of measure-preserving, orientation-preserving diffeomorphisms with no periodic points.

To formulate our results and place the comments above into our framework, we need some concepts from symplectic geometry. A smooth manifold is called symplectic if there exists a nondegenerate, closed, differentiable 2-form $\omega$ defined on $M$. A differentiable mapping $f$ of $M$ into itself is called symplectic if $f$ preserves the form $\omega$. We refer to the texts by Abraham and Marsden [A \& M] and Arnold [A] for the general background in symplectic geometry.

We reformulate Bourgin's question to ask: does every symplectic mapping of a $2 n$-dimensional cell, equipped with a symplectic structure, have a fixed point? Using a generalization of a theorem of Weinstein $\left[\mathbf{W}_{2}\right]$, we answer this question affirmatively for mappings sufficiently close to the identity.

Received by the editors January 22, 1981 and, in revised form, July 2, 1981; presented to the Society at the annual meeting in San Francisco, January 8, 1981.

1980 Mathematics Subject Classification. Primary 58D05; Secondary 53C15, 55M20, 70H15.

Key words and phrases. Symplectic manifold, fixed points, open manifold, symplectic diffeomorphism. 
1. Preliminaries. All manifolds are assumed to be finite-dimensional, $C^{\infty}$-smooth, and without boundary. A manifold $M$ is open if $M$ has no compact components. Let $\varepsilon(M)$ denote the ends of $M$, and let $\tilde{M}=M \cup \varepsilon(M)$ be the completion of $M$. We consider manifolds $M$ where the number of ends, denoted by $e(M)$, is finite and where $\tilde{M}$ has a smooth manifold structure without boundary. For the general problem of completing an open manifold with finitely many ends see Siebenmann's thesis [S].

If $M$ is a manifold with symplectic form $\omega$, then $\operatorname{Diff}(M, \omega)$ denotes the group of symplectic diffeomorphisms of $M$. The closed one-forms on $M$ are denoted by $Z^{l}(M)$. Both of these function spaces are topologized with the $C^{1}$-fine topology. See [H, p. 35] for a good account of the $C^{1}$-fine topology.

We require the basic formalism of "cotangent co-ordinates" contained in the following theorem of Weinstein.

Theorem 1.1 [ $\mathbf{W}_{1}$, Proposition (2.7.4) or $\mathbf{W}_{2}$, Theorem 7.2]. If $(M, \omega)$ is a symplectic manifold, then there is a $C^{1}$-fine neighborhood $A \subset \operatorname{Diff}(M, \omega)$ containing the identity map, $a C^{1}$-fine neighborhood $B \subset Z^{1}(M)$ containing the zero form, and $a$ homeomorphism $V: A \rightarrow B$. If $f \in A$, then a point $x \in M$ is a fixed point of $f$ if and only if $(V(f))(x)=0$.

Proof. If $f$ is in $\operatorname{Diff}(M, \omega)$, then the graph of $f$ is a Lagrangian submanifold of $M \times M$ with the symplectic structure $\pi_{1}^{*} \omega-\pi_{2}^{*} \omega$, where $\pi_{1}$ and $\pi_{2}$ are the projections. There exists a neighborhood $U$ of the diagonal $\Delta(M)=\{(m, m): m \in M\}$ and a bijection of $U$ onto a neighborhood $W$ of the zero-section in $T^{*} M$, taking Lagrangian submanifolds of $U$ onto Lagrangian submanifolds lying in $W$. If $f$ is close enough to the identity, in the sense that the graph of $f$ is contained in $U$, then there is a one-form $V(f) \in Z^{1}(M)$ whose image is contained in $W$. Clearly, $f(x)=x$ if and only if $(V(f))(x)=0$.

Various fixed point theorems in symplectic geometry result from Theorem 1.1. For examples see [ $\mathbf{M}, \mathbf{N}, \mathbf{S}, \mathbf{W}_{\mathbf{1}}$, and $\left.\mathbf{W}_{2}\right]$. Let $M$ be a compact manifold and $\eta$ a closed one-form. Define $c(\eta)$ to be the number of zeros of $\eta$. Define $c(M)=$ glb $\left\{c(\eta): \eta \in Z^{1}(M)\right\}$. If $M$ is a symplectic manifold with symplectic form $\omega$, then there is a $C^{1}$-neighborhood of $\operatorname{id}_{M}$ in $\operatorname{Diff}(M, \omega)$, so that if $f$ is in this neighborhood, then $V(f)$ is a closed one-form. Furthermore, the number of fixed points of $f$ is equal to $c(V(f))$. Now assume $M$ is simply connected, so that every closed one-form is exact. Then $c(M) \geqslant 2$ since every smooth function on a compact manifold has at least two critical points. Therefore, in this $C^{1}$-neighborhood of $\operatorname{id}_{M}$ every $f$ has at least two fixed points.

2. The main theorem. When the manifold $M$ is not compact there are functions with no critical points, and hence there are closed one-forms with no zeros. Therefore, $c(M)=0$. In this section we extend the fixed point theorem of Weinstein to open symplectic manifolds. Note that while $M$ may be a symplectic manifold, its completion $\tilde{M}$ may carry no symplectic structure at all. In particular, for the open 
$2 n$-cell $B^{2 n}=\left\{x \in \mathbf{R}^{2 n}:\|x\|<1\right\}$ the completion is homeomorphic to $S^{2 n}$, which has no symplectic structure for $n>1$. The open manifold $B^{2 n}$ has the standard symplectic structure induced from $\mathbf{R}^{2 n}$.

THEOREM 2.1. If $(M, w)$ is a symplectic manifold with $e(M)<c(\tilde{M})$, then there exists a $C^{1}$-fine neighborhood $A$ of $\operatorname{id}_{M}$ in $\operatorname{Diff}(M, \omega)$ such that every $f \in A$ has at least $c(\tilde{M})-e(M)$ fixed points.

Proof. Assume $M$ is embedded in $\tilde{M}$ as an open submanifold. Let $\phi: \tilde{M} \rightarrow \mathbf{R}$ be a nonnegative function vanishing only on the ends of $M, \phi(x)=0$ if and only if $x \in \tilde{M}-M$. Let $B \subset Z^{1}(M)$ be the set of one-forms defined by $\phi$,

$$
B=\left\{\eta \in Z^{1}(M):\|\eta(x)\|<\phi(x),\|D \eta(x)\|<\phi(x)\right\}
$$

where the norms arise from a riemannian metric on $\tilde{M}$. So $B$ is an open subset and every $\eta \in B$ extends to a form $\tilde{\eta}$ on $\tilde{M}$ such that $\tilde{\eta}(x)=0$ for $x \in \tilde{M}-M$. By taking an intersection, if necessary, we may assume that $B$ satisfies the conclusions of Theorem 1.1. Since $c(\tilde{M})-e(M)>0$ and $c(\tilde{\eta}) \geqslant c(\tilde{M})$, it follows that $c(\tilde{\eta}$. $e(M)>0$, so that $\tilde{\eta}$ has more zeros than there are points in $\tilde{M}-M$. Therefore $\eta(x)=0$ for some $x \in M$. Now we use Theorem 1.1 to get a $C^{1}$-fine neighborhood $A$ in $\operatorname{Diff}(M, \omega)$ containing the identity and a homomorphism $V: A \rightarrow B$. For $f \in A$, the one-form $V(f)$ is in $B$ and so $f$ has a fixed point $x$ in $M$.

We now restrict our attention to manifolds $M$ diffeomorphic to $\mathbf{R}^{2 n}$. Let $\omega$ be any symplectic structure on $M$. Clearly, $e(M)=1$ and by picking a point $N \in S^{2 n}$, we can embed $M$ onto $S^{2 n}-\{N\}$, so that $\tilde{M} \approx S^{2 n}$. With this construction and the fact that $c\left(S^{2 n}\right)=2$, we have

COROLlaRY 2.2. Let $(M, \omega)$ be a symplectic manifold where $M$ is diffeomorphic to $\mathbf{R}^{2 n}$. Then there is a neighborhood in the $C^{1}$-fine topology of $\operatorname{Diff}(M, \omega)$ which contains $\mathrm{id}_{M}$, such that every mapping in this neighborhood has a fixed point.

One should be aware that there are symplectic diffeomorphisms of $\mathbf{R}^{2 n}$ with symplectic structure $\Sigma d x_{i} \wedge d y_{i}$ that have no fixed points, in particular the translations, but there are $C^{1}$-fine neighborhoods of the identity containing no translations. Let $\phi: \mathbf{R}^{2 n} \rightarrow \mathbf{R}^{+}$be a function vanishing at infinity and use $\phi$ to define an open neighborhood consisting of the diffeomorphisms $f$ such that $\|f(x)-x\|<\phi(x)$ and $\|D f(x)-I\|<\phi(x)$ for all $x \in \mathbf{R}^{2 n}$.

\section{REFERENCES}

[A \& M] R. Abraham and J. E. Marsden, Foundations of mechanics, Benjamin/Cummings, Reading, Mass., 1978.

[A] V. I. Arnold, Mathematical methods of classical mechanics, Springer-Verlag, New York, 1978.

[As] D. Asimov, On volume preserving homeomorphisms of the open n-disk, Houston J. Math. 2 (1976), $1-3$.

[B] D. G. Bourgin, Homeomorphisms of the open disk, Studia Math. 31 (1968), 433-438.

[H] M. W. Hirsch, Differential topology, Springer-Verlag, New York, 1976.

[M] J. Moser, A fixed point theorem in symplectic geometry, Acta Math. 141 (1978), 17-34.

[N] N. Nikishin, Fixed points of diffeomorphisms on the two sphere that preserve area, Funkcional. Anal. i Priložen 8 (1974), 84-85. 
[S] L. Siebenmann, The obstruction to finding a boundary for an open manifold of dimension $\geqslant 5$, Thesis, Princeton Univ., Princeton, N.J., 1965.

[Si] C. P. Simon, $A$ bound for the fixed point index of an area preserving map with applications to mechanics, Invent. Math. 26 (1974), 187-200 and 32 (1976), 101.

$\left[\mathbf{W}_{1}\right]$ A. Weinstein, Periodic orbits of hamiltonian systems via critical point theory, manuscript, Univ. of California, Berkeley, 1971.

$\left[\mathbf{W}_{2}\right]$ , Lagrangian submanifolds and hamiltonian systems, Ann. of Math. (2) 98 (1973), 377-410.

Department of Mathematics, California Polytechnic State University, San luis Obispo, CALIFORNIA 93407 